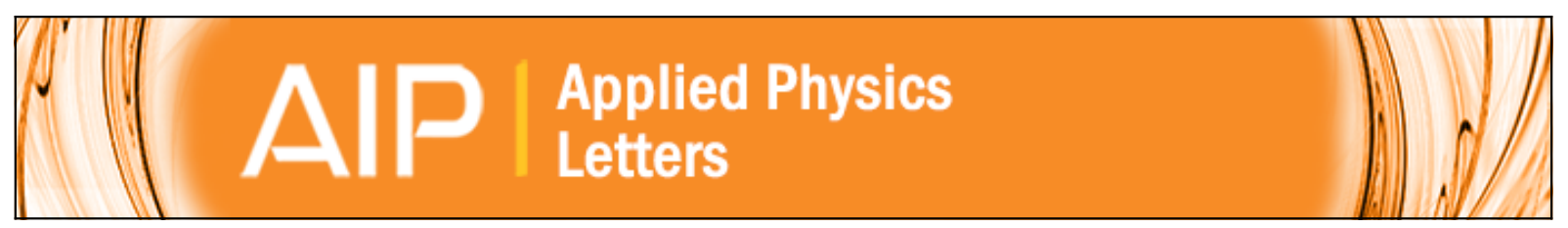

\title{
Field-effect active plasmonics for ultracompact electro-optic switching
}

Arif E. Çetin, Ahmet A. Yanik, Alket Mertiri, Shyamsunder Erramilli, Özgür E. Müstecaplolu, and Hatice Altug

Citation: Applied Physics Letters 101, 121113 (2012); doi: 10.1063/1.4754139

View online: http://dx.doi.org/10.1063/1.4754139

View Table of Contents: http://scitation.aip.org/content/aip/journal/apl/101/12?ver=pdfcov

Published by the AIP Publishing

\section{Articles you may be interested in}

A proposal for digital electro-optic switches with free-carrier dispersion effect and Goos-Hanchen shift in siliconon-insulator waveguide corner mirror

J. Appl. Phys. 114, 104502 (2013); 10.1063/1.4820378

Plasmon-induced transparency in metamaterials: Active near field coupling between bright superconducting and dark metallic mode resonators

Appl. Phys. Lett. 103, 101106 (2013); 10.1063/1.4819389

Plasmonic optical switches based on Mach-Zender interferometer

Phys. Plasmas 18, 072112 (2011); 10.1063/1.3614521

Electro-optic switching element for dielectric-loaded surface plasmon polariton waveguides

Appl. Phys. Lett. 97, 041107 (2010); 10.1063/1.3464552

Microring resonator based modulator made by direct photodefinition of an electro-optic polymer

Appl. Phys. Lett. 92, 153310 (2008); 10.1063/1.2908914

\section{NEW! Asylum Research MFP-3D Infinity ${ }^{\mathrm{m}}$ AFM}

Unmatched Performance, Versatility and Support

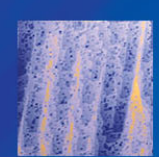

Comprehensive tools for nanomechanics
Stunning high performance
Simpler than ever to GetStarted ${ }^{\mathrm{TM}}$

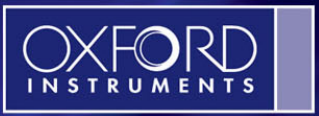

The Business of Science ${ }^{\mathrm{e}}$

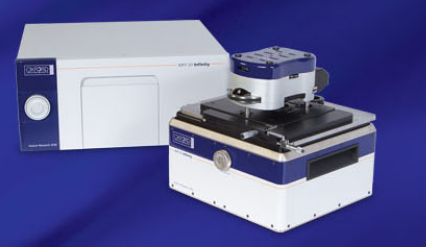




\title{
Field-effect active plasmonics for ultracompact electro-optic switching
}

\author{
Arif E. Çetin, ${ }^{1,2, a)}$ Ahmet A. Yanik, ${ }^{3, a)}$ Alket Mertiri, ${ }^{2,4}$ Shyamsunder Erramilli, ${ }^{2,4,5}$ \\ Özgür E. Müstecaplıoğlu, ${ }^{6}$ and Hatice Altug ${ }^{1,2,4, b)}$ \\ ${ }^{1}$ Department of Electrical and Computer Engineering, Boston University, Boston, Massachusetts 02215, USA \\ ${ }^{2}$ Boston University Photonics Center, Boston, Massachusetts 02215, USA \\ ${ }^{3}$ Center for Engineering in Medicine, Massachusetts General Hospital and Harvard Medical School, Boston, \\ Massachusetts 02129, USA \\ ${ }^{4}$ Division of Materials Science and Engineering, Boston University, Boston, Massachusetts 02215, USA \\ ${ }^{5}$ Department of Physics and Biomedical Engineering, Boston University, Boston, Massachusetts 02215, USA \\ ${ }^{6}$ Department of Physics, Koç University, Sariyer, Istanbul 34450, Turkey
}

(Received 21 August 2012; accepted 6 September 2012; published online 20 September 2012)

\begin{abstract}
Merging of electronics and photonics at subwavelength dimensions could potentially allow development of ultracompact electro-optic modulators and active optical interconnects. Here, we introduce a field-effect active plasmonic modulator where the metallic ring serves as both a photonic resonator and a field electrode. By exploiting the simultaneous electronic and photonic functionalities of our plasmonic device, we show devices offering significantly improved modulation depths (as high as $\sim 10.85 \mathrm{~dB}$ ) compared to active dielectric micro-ring resonators. Device concepts introduced in this work are applicable in realization of various integrated components and could play an important role in development of active plasmonic circuits. (C) 2012 American Institute of Physics. [http://dx.doi.org/10.1063/1.4754139]
\end{abstract}

Recent advancements in nanofabrication capabilities enabling integration of electronic and photonic components have opened up a wide range of opportunities in creation of systems with multiple functionalities. In particular, on-chip optical interconnects offering extremely large bandwidths and reduced power consumptions have taken much interest due to their potential to circumvent the RC signal delay bottleneck in electronic circuits. ${ }^{1-4}$ However, after four decades of miniaturization efforts, convergence of these two complementary technologies seems to be hindered. A fundamental constraint is the size-compatibility problem. Minimum feature sizes in the photonic devices are restricted to micron dimensions by light's diffraction limit, making them bulkier than the nano-electronic components. Innovative approaches are needed to overcome this size compatibility issue. By confining electromagnetic fields to sub-wavelength dimensions as small as few tens of nanometers, surface plasmonics offers a route to bridge the dimension gap between electronics and photonics. ${ }^{5-12}$ Within the last decade, preliminary demonstrations of active plasmonic devices exploiting the propagation characteristics of the surface plasmon and nonlinear materials have been shown. ${ }^{3,13,14}$ Possibility of such plasmonic devices that can act as actively controlled interconnects is extremely promising. However, plasmonic modulators and switches that are considered so far require dimensions at micron-scales due to the weak nonlinear optical effects. ${ }^{3,13}$ Furthermore, practically large modulation depths have yet to be demonstrated. ${ }^{15}$

In this letter, we introduce an ultra-compact metallic ring device utilizing actively controlled plasmonic resonances by field effects for strong electro-optic modulation of electromagnetic signals at subwavelength dimensions. A

\footnotetext{
a) A. E. Çetin and A. A. Yanik contributed equally to this work.

b) Author to whom correspondence should be addressed. Electronic mail: altug@bu.edu.
}

schematic diagram of the proposed field effect active plasmonic ring modulator is shown in Fig. 1(a). Unlike widely adapted photonic micro-ring modulators (shown in Fig. 1(b)), our device takes advantage of the metallic nature of the optical resonator, where the ring serves both as a photonic resonator and an electrode. ${ }^{11}$ This unique capability enables three major advancements over standard micro-ring resonators: (i) Dual use of metallic ring both as a resonator and as an electrode allows creation of large modulation fields right at the device surfaces where the electro-magnetic signals are confined. (ii) The radial symmetry of the central electrodes in our modulator configuration offers stronger dynamic tuning of the ring resonances by creating larger on/ off effective refractive index differences than that of standard double-electrode configuration used in micro-ring

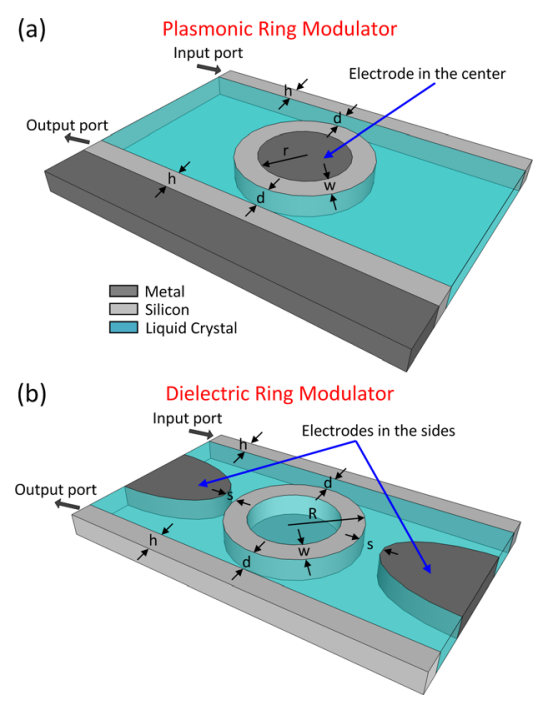

FIG. 1. Schematic diagrams of (a) plasmonic ring modulator where the electrode is located in the center of the system and (b) dielectric ring modulator where the electrodes are located in the right and left sides of the ring. 
resonators. ${ }^{16}$ (iii) The nano-scale dimensions of our ringresonator lead to much larger electric fields for stronger electro-optic modulation than conventional micro-ring counterparts. ${ }^{16}$ As we show in the following, these capabilities enabled by our unique electrode-resonator configuration could open doors to devices with large modulation depths at low voltages compatible with silicon electronics.

The plasmonic ring modulator studied in this letter consists of a metallic core with a radius of $r=720 \mathrm{~nm}$ and a silicon dielectric ring with a thickness of $w=280 \mathrm{~nm}$ around it. The resonator is located between two dielectric waveguides at a gap distance $d=40 \mathrm{~nm}$. The dielectric ring around the metallic core is critical for suppression of the radiative losses leading to high quality factor resonances as large as $Q=76$ even at subwavelength dimensions. ${ }^{17}$ This dielectric ring also allows efficient in-coupling of the electromagnetic field from the silicon dielectric waveguide at the top (width, $h=290 \mathrm{~nm}$ ) to the plasmonic ring resonator and out-coupling of the surface plasmon excitations from the metallic resonator to the plasmonic waveguide. A silicon dielectric waveguide at the bottom with identical width $(w)$ is also incorporated for efficient coupling of plasmons from the metallic core to the output port that can interface with nano-electronic components. Electrical modulation of the effective refractive index of the plasmonic ring resonator is needed for dynamic tuning of resonances. This is achieved by using liquid crystals filling the medium between the waveguides and the metallic core constituting the resonator/electrode. ${ }^{18,19}$ Liquid crystals have very high contrast between extraordinary and ordinary refractive indices even though their electro-optic response is not fast. We used a commercially available liquid crystal, E63, as the dynamic tuning medium. Ordinary $\left(n_{o}\right)$ and extraordinary $\left(n_{e}\right)$ refractive indices of E63 are 1.517 and 1.744, respectively. In the absence of electrode voltage, it is assumed that the local directors of liquid crystal are completely random resulting in a cladding refractive index, $n_{\text {clad }}=1.596 .{ }^{16}$ Reversible effective refractive index modulation is achieved by preferential orientation of the local directors along the direction of the applied electric field. For comparison purposes, a dielectric ring resonator with identical dimensions and similar free spectral range is also included in our analysis (Fig. 1(b)). ${ }^{16}$ In this configuration, the electro-optic modulation is achieved by using two metallic electrodes located on the either side of the resonator with a gap distance of $s=200 \mathrm{~nm}$.

Fig. 2 compares the modulation capability of the radial electrode configuration offered by plasmonic ring modulator to that of the conventional dielectric one with double electrode arrangement. Calculations are performed using finiteelement method (FEM). At sufficiently strong electric fields above the saturation field $\left(E_{s a t}=0.52 \mathrm{~V} / \mu \mathrm{m}\right.$ for E63 (Ref. 16)), liquid crystal local directors are oriented along the direction of the static electric field lines to minimize their electrostatic energy. As we show in the following, this saturation condition is in fact readily met at low voltages compatible with silicon electronics for the metallic ring resonators. In Figs. 2(a) and 2(b), arrows indicate the direction of the static electric field due to the applied electrode voltages. In the case of cylindrically symmetric ring resonators, the oscillating electric field component of the surface plasmonic excitations or the photonic waveguide modes are
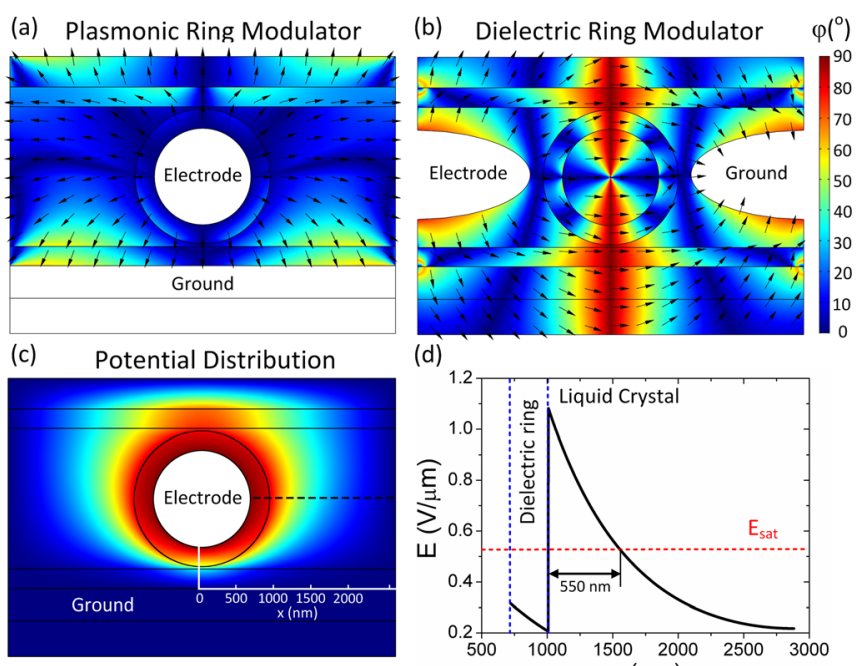

(d)

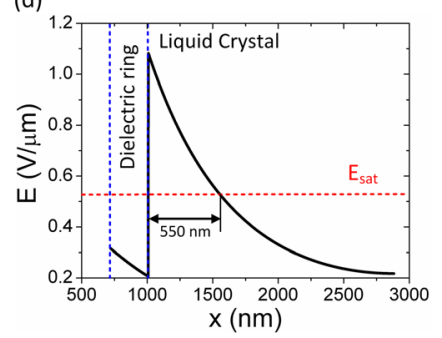

FIG. 2. Angular difference between the directors of the liquid crystal molecules and the radial axis for (a) plasmonic ring modulator with central electrode and (b) conventional dielectric ring modulator with double electrodes. In figures, arrows indicate the direction of the electric field generated by the electrodes. (c) Potential distribution of the plasmonic ring modulator system where the applied voltage from the electrode is $0.7 \mathrm{~V}$. (d) E-field distribution generated by the electrode along the $x$-axis. The data is taken along the black dashed line indicated in (c). In (d), saturation field for E63 is indicated by a red dashed line. The corresponding geometrical parameters are $r=720 \mathrm{~nm}$, $w=280 \mathrm{~nm}, h=290 \mathrm{~nm}, d=40 \mathrm{~nm}$, and $s=200 \mathrm{~nm}$.

polarized in the radial direction. Accordingly, color-scale shows the angular difference $(\varphi)$ between the local directors of the liquid crystals (parallel to the static electric field lines) and the radial axis of the ring resonators (corresponding to electromagnetic field polarization). In plasmonic ring devices (Fig. 2(a)), the applied electric field orienting the local directors along the direction of radial axis $(\varphi \approx 0$ throughout the ring structure) yields a strong modulation of the effective refractive index from that of the completely random distribution of local directors. On the other hand, in the conventional dielectric ring modulators with double electrode arrangement, tuning is only due to the partial alignment of liquid crystal directors and the polarization of the electromagnetic excitations (in radial direction). As shown in color scale (Fig. 2(b)), the angular difference in between changes from $\varphi \approx 0$ (blue in color-scale) to $\varphi \approx 90$ (red in color-scale) throughout the ring depending on the angular position relative to the axis of the electrodes. ${ }^{16}$ Accordingly, much weaker refractive index modulations for the devices incorporating conventional double electrodes configuration are expected. Furthermore, the conventional double electrode configuration suffers from extreme sensitivity to the spatial arrangement of the fabricated electrodes as shown in previous experiments, where much weaker modulations of cladding refractive index with respect to analytical predictions were observed. ${ }^{16}$ Another crucial advantage of plasmonic ring modulator is its capability to create large modulation fields right at the ring surfaces where the electromagnetic signals are confined. As a result, sufficiently strong electric fields saturating the local director orientations can be achieved at the close vicinity of the ring surface. Therefore, our plasmonic ring modulator is tolerant to the arrangement of the electrodes due to its radial symmetry. Fig. 2(c) shows 
the potential distribution of the plasmonic ring modulator where the applied potential at the central electrode is $0.7 \mathrm{~V}$. As shown in Fig. 2(d), for small applied voltages $(0.7 \mathrm{~V})$ compatible with silicon electronics, complete alignment of the liquid crystal directors is expected for distances about $550 \mathrm{~nm}$ from the dielectric ring surrounding the metallic core. Accordingly, this saturation region readily covers the extent of the surface plasmons confined to the resonators and enables strong modulation of the resonance wavelengths. In comparison, large voltages as high as $20 \mathrm{~V}$ were not sufficient for complete alignment of the liquid crystal directors in dielectric micro-ring resonators utilizing two electrode configurations, as shown in previous experimental studies. ${ }^{16}$

The on/off signal ratios are the core device characteristics for optical modulators in information technology applications. Transmission spectra of the plasmonic ring modulator under no $(V=0)$ and saturation voltage $\left(V=V_{\text {sat }}\right)$ are compared in Fig. 3(a). In our FEM calculations, an illumination source of TE-polarized Gaussian beam (electric field is in the plane of the resonator) is used to excite the waveguide modes through the input port as indicated in Fig. 1(a). The transmission characteristic of the system is determined by analyzing the intensity of the detected beam at the output port (Fig. 1(a)). Input and output ports are located $1.8 \mu \mathrm{m}$ away from the center of the ring resonators in horizontal axis. The dielectric properties of the metal are incorporated using a Drude model: $\varepsilon_{m}=1-\omega_{p}^{2} /\left(\omega^{2}+i \Gamma \omega\right)$ with parameters $\omega=9 \mathrm{eV}$ and $\Gamma=0.1 \mathrm{eV}$ following previous studies. ${ }^{17}$ Spatially inhomogeneous permittivity tensor of the liquid crystal molecules is determined by the electrostatic field lines, assuming that the applied electrode field is strong
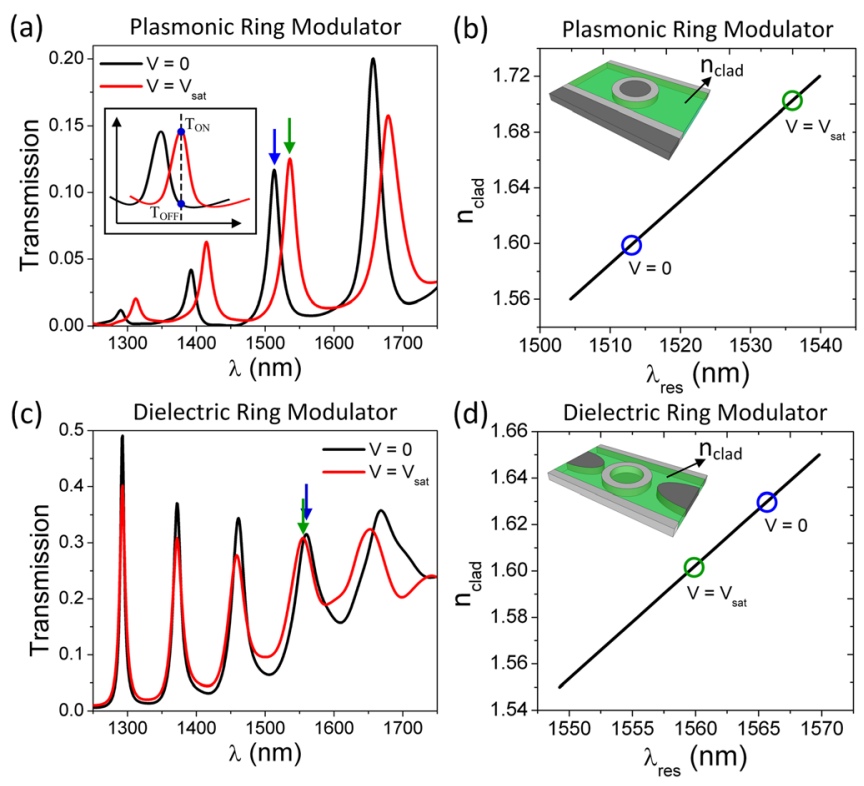

FIG. 3. Power transmission as a function of wavelength for (a) plasmonic and (c) dielectric ring modulators under no voltage (black curve) and saturation voltage, $V_{\text {sat }}$ (red curve). In the figure, blue and green arrows indicate the center wavelength of analyzed resonant modes within the telecommunication window under no voltage and saturation voltage, respectively. In the inset of (a), $T_{O N}$ and $T_{O F F}$ location is schematically illustrated. Refractive index of the isotropic cladding medium, $n_{\text {clad }}$, that corresponds to the center wavelength $\left(\lambda_{\text {res }}\right)$ of the resonator mode for (b) plasmonic and (d) dielectric ring modulators. The corresponding geometrical parameters are $r=720 \mathrm{~nm}$, $w=280 \mathrm{~nm}, h=290 \mathrm{~nm}, d=40 \mathrm{~nm}$, and $s=200 \mathrm{~nm}$. enough for the complete alignment of the local liquid crystal directors. In Fig. 3(a), multiple resonances are observed in the transmission spectra due to the resonance conditions corresponding to $m$ th resonator mode $(m=1,2,3$, .) defined by the phase relationship: $\lambda_{m}=2 \pi R n_{\text {eff }} / \mathrm{m}$, where $R$ is the radius of the resonator and $n_{\text {eff }}$ is the effective refractive index of the waveguide mode. As the effective refractive index increases by altering liquid crystal alignment from random orientation to the electric field direction, red shifting of the resonances is observed. In this letter, we focus on the transmission resonance at $\lambda_{\text {res }}=1513 \mathrm{~nm}$ (indicated by a blue arrow in Fig. 3(a)) within the telecommunication wavelength regime. For sufficiently large applied electrode potential saturating the local directors, the resonance wavelength shifts to $1536 \mathrm{~nm}$ (indicated a by green arrow), corresponding to a resonance wavelength modulation as large as $23 \mathrm{~nm}$. Effective refractive index of the waveguide mode $\left(n_{\text {eff }}\right)$ depends on the refractive indices of the cladding medium $\left(n_{\text {clad }}\right)$ and the dielectric ring (silicon). To calculate cladding refractive index, we represent liquid crystal with a homogeneous dielectric constant and obtain the values (blue and green circles in Fig. 3(b)) which give the corresponding resonances (blue and green arrows in Fig. 3(a)) in transmission spectrum. For our plasmonic device, an effective refractive index variation as large as $\Delta n_{\text {clad }}=0.102$ is observed due to the efficient reorientation of liquid crystal directors from random (at $V=0$, blue circle) to complete alignment (at $V=V_{\text {sat }}$, green circle). Fig. 3(c) shows the transmission spectra of the dielectric ring modulator of identical geometry. The structure supports five modes within the given spectral window. Here, we particularly focus on the transmission resonance centered at $1566 \mathrm{~nm}$ as it gives the largest modulation depth (modulation depth definition will be given in the next section). This mode with applied saturation voltage only shifts $6 \mathrm{~nm}$, corresponding to a smaller effective refractive index variation of only $\Delta n_{\text {clad }}=0.024$, as shown in Fig. 3(d). Accordingly, plasmonic ring modulator supports more than four times larger modulation in effective refractive index of the cladding medium under saturation conditions, which can be readily achieved at low voltages compatible with silicon electronics compared to the dielectric ring modulator. Interestingly, we observe that for the plasmonic ring modulator, the transmission resonance red-shifts with applied voltage whereas for the dielectric ring modulator, it blue-shifts. This blue-shift has been experimentally demonstrated in earlier works. ${ }^{16}$ For the dielectric system, liquid crystal directors are oriented azimuthally by the generated electric fields. Although, the refractive index is higher (close to $n_{e}$ ) for the regions close to electrodes (blue in color scale), azimuthal alignment of local directors leads to a drop in effective refractive index (towards $n_{o}$ ) at spatial locations away from the electrodes (red in color scale). When numerically averaged for an electromagnetic mode over the ring surface, the effective refractive index of the cladding medium $\left(n_{\text {clad }}\right)$ is relatively lower than the isotropic refractive index of the liquid crystal medium which causes the effective refractive index of the waveguide mode $\left(n_{\text {eff }}\right)$ to decrease. Consequently, the transmission resonance shifts to shorter wavelengths. In contrast, for the plasmonic ring modulator, the liquid crystal directors are well-aligned with the radial axis of the ring 
resonator (polarization direction of the electromagnetic modes) as demonstrated by the blue color scale. Such reorientation results in an increase of effective refractive index of the cladding medium towards $n_{e}$ so that the applied voltage leads to a red-shift in the transmission resonance.

In order to compare the modulation capability of the two ring modulator systems, we determine the modulation depth achieved under saturation condition. Here, modulation depth is calculated using $M D(d B)=10 \quad \log [1 /(1-\Gamma)]$, where $\Gamma=\left(T_{O N}-T_{O F F}\right) / T_{O N}$ and $T_{O N}\left(T_{O F F}\right)$ is the transmittance between in/output-ports at the resonance wavelength corresponding to complete (random) alignment of the liquid crystal with the applied voltage. The location of $T_{O N}$ and $T_{O F F}$ is schematically illustrated in the inset of Fig. 3(a). For the plasmonic ring modulator, the values of $T_{O N}$ and $T_{O F F}$ are 0.1254 and 0.0103 , corresponding to strong modulation depths as large as $10.85 \mathrm{~dB}$. However, the conventional dielectric configuration of identical geometry supports a much smaller modulation depth, only $0.42 \mathrm{~dB}$ for the analyzed transmission resonance centered at $1566 \mathrm{~nm}$. Such an improvement in the modulation depth observed for the plasmonic device is associated to large refractive index modulation capability enabled by the simultaneous photonic functionalities and radial symmetry of the metallic electrode. Unlike the dielectric systems, plasmonic devices suffer from radiative and nonradiative losses, which result in broadening of the resonances. ${ }^{20,21}$ For the plasmonic ring resonator system, numerically analyzed transmission resonance has a line-width as large as $20 \mathrm{~nm}$ that could negatively affect the modulation depth. However, this broadening effect is compensated with larger resonance shifts under saturation condition, which yields higher modulation depths. In contrast, for the conventional dielectric configuration, even though the transmission resonances are narrower, the electrode configuration is not capable to effectively orient the liquid crystal directors along the radial axis. Therefore, they suffer from weak refractive index modulation of cladding medium and have lower modulation depths. Performance of plasmonic ring modulator could be further improved with the use of alternative metallic materials promising good optical properties and low-losses. $^{22}$

In conclusion, we have introduced an active plasmonic modulator merging plasmonic waveguide and field electrode configuration into a single ring resonator structure. We have shown that our device enables creation of large modulation fields right at the metal surfaces where the signals are confined.
Radial symmetry of the central electrode and strong electric fields create large on/off effective refractive index differences. Therefore, the system enables stronger dynamic tuning of the ring resonances for stronger electro-optic modulation at low voltages compatible with silicon electronics. This unique scheme of exploiting surface plasmons also results in ultracompact device dimensions. Beyond the proposed ultracompact electro optic modulator design, the concepts introduced in this letter can lead to development of other integrated components merging electronics and photonics at nanoscale dimensions.

This research was supported by the Office of Naval Research (11PR00755-00-P00001), the National Science Foundation (ECCS-0954790), and the Massachusetts Life Sciences Center. Ö.E.M. acknowledges support by TUBITAK under Project No. 111T285.

\footnotetext{
${ }^{1}$ G. Chen, H. Chen, M. Haurylau, N. Nelson, P. M. Fauchet, E. G. Friedman, and D. Albonesi, in Proceedings of the 2005 International Workshop on System Level Interconnect Prediction, San Francisco, California, USA, 2005 (ACM, New York, NY, 2005), pp. 13-20.

${ }^{2}$ W. Cai, J. S. White, and M. L. Brongersma, Nano Lett. 9, 4403 (2009).

${ }^{3}$ J. A. Dionne, K. Diest, L. A. Sweatlock, and H. A. Atwater, Nano Lett. 9, 897 (2009).

${ }^{4}$ S. Sandhu and S. Fan, Opt. Express 20, 4280 (2012).

${ }^{5}$ E. Ozbay, Science 311, 189 (2006).

${ }^{6}$ K. Aydin, V. E. Ferry, R. M. Brigss, and H. A. Atwater, Nat. Commun. 2, 517 (2011).

${ }^{7}$ A. L. Lereu, A. Passian, J. P. Goudonnet, T. Thundat, and T. L. Ferrell, Appl. Phys. Lett. 86, 154101 (2005).

${ }^{8}$ A. V. Krasavin and N. I. Zheludev, Appl. Phys. Lett. 84, 1416 (2004).

${ }^{9}$ S. Randhawa, A. V. Krasavin, T. Holmgaard, J. Renger, S. I. Bozhevolnyi, A. V. Zayats, and R. Quidant, Appl. Phys. Lett. 98, 161102 (2011).

${ }^{10}$ E. Cubukcu, N. Yu, E. J. Smythe, L. Diehl, K. B. Crozier, and F. Capasso, IEEE J. Sel. Top. Quantum Electron. 14, 1448 (2008).

${ }^{11}$ R. Zia, A. Chandran, and M. L. Brongersma, Opt. Lett. 30, 1473 (2005).

${ }^{12}$ K. Guven and B. Tanatar, Phys. Rev. B 56, 7535 (1997).

${ }^{13}$ K. F. MacDonald, Z. L. Samson, M. I. Stockman, and N. I. Zheludev, Nat. Photonics 3, 55 (2009).

${ }^{14}$ T. Nikolajsen, K. Leosson, and S. I. Bozhevolnyi, Appl. Phys. Lett. 85, 5833 (2004).

${ }^{15}$ R. A. Pala, K. T. Shimizu, N. A. Melosh, and M. L. Brongersma, Nano Lett. 8, 1506 (2008).

${ }^{16}$ B. Maune, R. Lawson, C. Gunn, A. Scherer, and L. Dalton, Appl. Phys. Lett. 83, 4689 (2003).

${ }^{17}$ B. Wang and G. P. Wang, Appl. Phys. Lett. 89, 133106 (2006).

${ }^{18}$ A. E. Cetin and O. E. Mustecaplioglu, Phys. Rev. A 81, 043812 (2010).

${ }^{19}$ F. S. Collins, M. Morgan, and A. Patrinos, Science 300, 286 (2003).

${ }^{20}$ T. Kokkinakis and K. Alexopoulos, Phys. Rev. Lett. 28, 1632 (1972).

${ }^{21}$ S. Link and M. A. El-Sayed, Int. Rev. Phys. Chem. 19, 409 (2000).

${ }^{22}$ D. A. Bobb, G. Zhu, M. Mayy, A. V. Gavrilenko, P. Mead, V. I. Gavrilenko, and M. A. Noginov, Appl. Phys. Lett. 95, 151102 (2009).
} 